\title{
The Letter to the Editor regarding the paper'Evaluation of the efficiency of fluoride-releasing adhesives for preventing secondary caries in-vitro: a systematic review and meta-analysis'
}

\author{
F. V. Martins ${ }^{1}$ (D) - W. F. Vasques ${ }^{1} \cdot$ E. M. Fonseca ${ }^{2}$
}

Received: 14 June 2019 / Accepted: 19 June 2019 / Published online: 25 June 2019

c) European Academy of Paediatric Dentistry 2019

I would like to thank the European Archives of Paediatric Dentistry and Drs Cuncun Lu and Ming Liufor, for the letter and the opportunity.

As Moher et al. 2009 state: "Systematic reviews and meta-analyzes have become increasingly important in health care. Clinicians read them to keep up with a date with their field, and they are often used as a starting point for developing clinical practice guidelines .........

In their study Moher et al. (2009) 'Preferred reporting items for systematic reviews and meta-analyses: the PRISMA statement'. Table 1, Section/Topic \# 8, it is stated: "Present full electronic search strategy for at least one database, including any limits used, such that it could be repeated."

In our paper Martins et al. 2019, we informed the full electronic search strategy, so it could be repeated. We understand that for this purpose it is necessary that the reader has a minimum understanding about the systematic review or a minimum understanding of how to use the search bases.

However, one of our goals is to facilitate the reading for new beginners. Therefore, we would like to thank for the opportunity and make the following clarification shown below:

Search strategy (the addition and clarification to the initial publication is shown underlined)

The bibliographic search was carried out in the following search bases: MEDLINE - National Library of Medicine via PubMed, Web of Science and Scopus databases to identify relevant articles between January 2000 and September 2017. The specific medical subject headings

F. V. Martins

felipe37451@gmail.com

1 College of Dentistry, Federal Fluminense University (UFF), Mario Santos Braga St., 28-Centro, Niterói, RJ 24020-140, Brazil

2 Department of Dental Technique, Federal Fluminense University (UFF), Niterói, RJ, Brazil
(MeSH) and the key words (free text word) were also used. The searches were carried out independently by two authors, using the following research strategy for MEDLINE/PubMed: (()(()(()(()(()((Leakage[MeSH Terms]) OR restoration[MeSH Terms]) OR adhesives[MeSH Terms]) OR bonding[MeSH Terms]) OR bond[MeSH Terms]) OR caries[MeSH Terms]) OR secondary caries[MeSH Terms]) OR Leakage[Title/Abstract]) OR restoration[Title/ Abstract]) OR adhesives[Title/Abstract]) OR bonding[Title/ Abstract]) OR bond[Title/Abstract]) OR caries[Title/ Abstract]) OR secondary caries[Title/Abstract])) AND ((()((cariostatic[MeSH Terms]) OR fluorides[MeSH Terms]) OR fluoride[MeSH Terms]) OR cariostatic[Title/ Abstract]) OR fluorides[Title/Abstract]) OR fluoride[Title/ Abstract])) AND ((((Remineralization[MeSH Terms]) OR demineralization[MeSH Terms]) OR Remineralization[Title/ Abstract]) OR demineralization[Title/Abstract])) AND ((( (in-vitro[MeSH Terms]) OR in vitro[MeSH Terms]) OR in-vitro[Title/Abstract]) OR in vitro[Title/Abstract]).

Best regards

Felipe Villela Martins

\section{References}

Martins FV, Vasques WF, Fonseca EM. Evaluation of the efficiency of fluoride-releasing adhesives for preventing secondary caries invitro: a systematic review and meta-analysis. Eur Arch Paediatr Dent. 2019;20(1):1-8.

Moher D, Liberati A, Tetzlaff J, et al. Preferred reporting items for systematic reviews and meta-analyses: the PRISMA statement. BMJ. 2009. https://doi.org/10.1136/bmj.b2535s.

Publisher's Note Springer Nature remains neutral with regard to jurisdictional claims in published maps and institutional affiliations. 